\title{
Poisson versus periodic path probing (or, does PASTA matter?)
}

\author{
Muhammad Mukarram Bin Tariq, Amogh Dhamdhere, Constantinos Dovrolis, Mostafa Ammar \\ Georgia Institute of Technology \\ \{mtariq,amogh,dovrolis,ammar\}@cc.gatech.edu
}

\begin{abstract}
The well-known PASTA ("Poisson Arrivals See Time Averages") property states that, under very general conditions, the fraction of Poisson arrivals that observe an underlying process in a particular state is equal, asymptotically, to the fraction of time the process spends in that state. When applied to network inference, PASTA implies that a Poisson probing stream provides an unbiased estimate of the desired time average. Our objective is to examine the practical significance of the PASTA property in the context of realistic RTT, loss rate and packet pair dispersion measurements with a finite (but not small) number of samples. In particular, we first evaluate the differences between the point estimates (median RTT, loss rate, and median dispersion) that result from Poisson and Periodic probing. Our evaluation is based on a rich set of measurements between 23 PlanetLab hosts. The experimental results show that in almost all measurement sessions the differences between the Poisson and Periodic point estimates are insignificant. In the case of RTT and dispersion measurements, we also used a non-parametric goodness-of-fit test, based on the Kullback-Leibler distance, to evaluate the similarity of the distributions that result from Poisson and Periodic probing. The results show that in more than $90 \%$ of the measurements there is no statistically significant difference between the two distributions.
\end{abstract}

\section{Introduction}

In the context of active measurements, a sequence of probing packets is injected in a network path with the objective to measure end-to-end properties such as RoundTrip Time (RTT), loss rate, or available bandwidth (related to the time-spacing, or dispersion, between successive packets). A central issue in active measurements is the characteristics of the probing process [1]. From a mathematical perspective, it is often claimed that "the right way" to do probing (or sampling) is to use a Poisson process (meaning that the interarrivals between probing packets should be independent and exponentially distrib- uted with the same mean) $[2,3,4,5,6]$.

Poisson probing derives its strength from the wellcited PASTA property, which stands for "Poisson Arrivals See Time Averages" [7]. Consider a stochastic system and suppose that we want to infer the fraction of time $p_{X}$ the system spends in a state $X$. Let us assume that this time average exists. A stream of $N$ "probes" arrives in the system based on a Poisson process, recording the system's state at the arrival time instant. PASTA states that the expected value $E\left[f_{X, N}\right]$ of the fraction of Poisson probes that observe the system at state $X$ is equal to the time average $p_{X}$. This powerful result does not make any assumptions about the stochastic system, except that the time average $p_{X}$ exists; stationarity or ergodicity may be needed in order to prove that however.

The underlying reason for the validity of PASTA is that the monitored system cannot "anticipate" the next probing event, due to the memoryless nature of the Poisson measurement process. Notice that the the Poisson probes may be interacting with the monitored system. In the case of a queueing system, for example, probing packets can be backlogged, serviced, or dropped, as any other packet, and they may also affect the magnitude of the time average $p_{X}$. It is also important to note that PASTA is an asymptotic result: $f_{X, N}$ tends to $p_{X}$ as the number of probes $N$ tends to infinity. The sample average $f_{X, N}$, even though an unbiased estimate, can significantly deviate around $p_{X}$ depending on $N$ and on the variability and correlation structure of the underlying system [8].

On the practical side, the most common measurement approach is to use Periodic probing, rather than Poisson probing. For instance, the popular ping utility generates a periodic probing stream. An important advantage of Periodic probing is that the duration of a measurement session can be a priori known, given the number of samples and the probing period. Furthermore, scheduling periodic packet transmissions at mainstream (not realtime) operating systems is easier and more accurate than 
scheduling random, and potentially very short or very long, packet interarrivals. It should be noted that the use of Periodic probing does not mean that the resulting estimate will be necessarily biased, especially if the probing rate is sufficiently high. It is true, however, that only Poisson probing can provably result in an unbiased estimate under general conditions.

An important question, which is still unresolved to the best of our knowledge, is whether PASTA "matters" in the pragmatic context of RTT, loss rate, and bandwidth (or dispersion) measurements in the Internet. Given that Periodic probing has some important practical benefits, we need to know whether Poisson and Periodic probing sequences lead to significantly different estimates. In this paper, we focus on three significant path performance metrics: RTT, loss rate and dispersion of back-to-back packet pairs. Our objective is to evaluate the differences that result from Poisson and Periodic probing, both in terms of point estimates for the first moment of the underlying distributions, as well as in terms of differences in the distributions themselves. Note that, since this is a measurement study over wide-area Internet paths, we do not know whether Poisson and/or Periodic probing measure the actual time average of the previous performance metrics. Instead, we can only examine whether the two probing techniques observe the same (but potentially wrong!) path performance.

In Section 2, we describe our measurement collection process. In Sections 3, 4, and 5 we analyze the RTT, loss rate, and dispersion measurements, respectively. Our results show that in almost all measurement sessions the differences between Poisson and Periodic point estimates are insignificant. In the case of RTT and dispersion measurements, there is no statistically significant difference between the Poisson and Periodic distributions in about $90 \%$ of the measurements. We conclude with some additional remarks in Section 6.

\section{Measurement methodology}

We collected measurements of RTT, packet loss rate, and packet pair dispersion in network paths between 23 PlanetLab hosts. Specifically, we used PlanetLab nodes at the following sites: RNP (Brazil), UC San Diego, UC Berkeley, U-Oregon, U-British Columbia, UTexas, UIUC, Georgia Tech, CMU, MIT, U-Maryland, INRIA (France), Intel Research Cambridge (UK), UPC (Spain), U-Reykjavik (Iceland), EPFL (Switzerland), Helsinki Inst. of Tech. (Finland), U-Tsinghua (China), Hong Kong Univ. of Science \& Tech, National Taiwan Univ, Equinix (Singapore), U-Tokyo (Japan), and U-Melbourne (Australia). The measurements reported in this paper were collected from 53 source-destination pairs. Each measurement session (or simply "session") consists of 600 Poisson probes and 600 Periodic probes transmitted at the same average rate. The two probing streams of a session start at the same time, and so they cover approximately the same time window.

A session is characterized by a source-destination pair, the average probing interarrival $I$, and the packet size $L$. The probing interarrival was $10 \mathrm{~ms}, 20 \mathrm{~ms}, 50 \mathrm{~ms}, 100 \mathrm{~ms}$, $500 \mathrm{~ms}$, or $1 \mathrm{sec}$, meaning that a session lasts from $6 \mathrm{sec}-$ onds to 10 minutes, depending on $I$. For the RTT and loss rate sessions $L$ was $32,64,480$, or 1400 bytes, while for the dispersion measurements $L$ was 480, 800, or 1400 bytes. The number of successfully completed sessions was 1272 for each of the RTT and loss rate measurements and 954 for the dispersion measurements.

It turned out that some paths were either too slow for our probing streams (especially with $I=10 \mathrm{~ms}$ or $20 \mathrm{~ms}$ and large packet sizes), or they were extremely congested. In a pre-processing step, we filtered out all sessions in which the loss rate was higher than $10 \%$ in the RTT and dispersion analysis; those sessions were included, however, in certain parts of the loss rate analysis (as noted in $\S 4$ ). The number of sessions we were left with was 892 for RTT and loss rate and 749 for dispersion. The RTT and dispersion time measurements were obtained with kernel-level (libpcap) timestamps, reported in a resolution of one microsecond.

\section{RTT measurements}

Comparison of median RTTs: The sample median is a robust point estimate for the first moment of a distribution. Let $T_{e}$ and $T_{p}$ be the median RTTs estimated from the Poisson and Periodic probing streams, respectively, in a particular session. We define the relative difference between the two RTT medians as $\epsilon_{T}=2 \frac{T_{e}-T_{p}}{T_{e}+T_{p}}$. Figure 1 shows the empirical CDF of $\epsilon_{T}$. About $60 \%$ of the sessions do not see any difference $\left(\epsilon_{T} \approx 0\right)$, while about $85 \%$ of the sessions have $\left|\epsilon_{T}\right|<1 \%$. The maximum relative difference is $4 \%$. The results are actually quite similar for the relative difference of RTT means. Consequently, at least in relative error terms, Poisson and Periodic probing result in practically the same RTT estimate. The fact that the two probing processes measure almost equal median RTTs, however, does not mean that they also observe the same RTT distribution; we examine this issue next.

Goodness-of-fit test: To further explore the differences between Poisson and Periodic probing, we also examine the RTT distributions measured by the two probing processes. Let $S_{e}$ and $S_{p}$ be the Poisson and Periodic RTT samples, respectively, collected in a particular session. We form the following null hypothesis:

$$
H_{0}: S_{e} \text { and } S_{p} \text { follow identical distributions. }
$$

The alternate hypothesis is that there is a statistically significant difference between the two distributions. A 


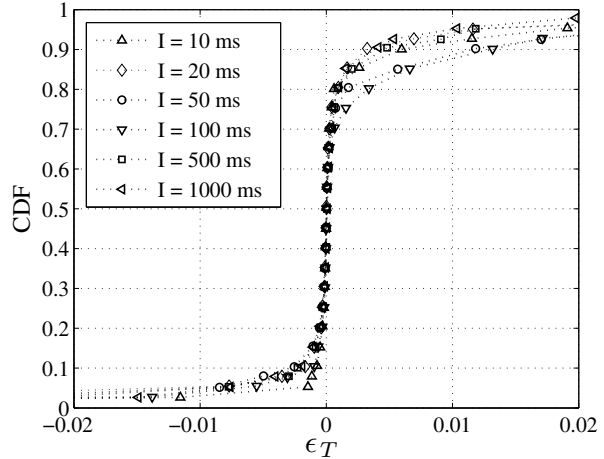

Figure 1: The relative difference $\epsilon_{T}$ between Poisson and Periodic RTT medians.

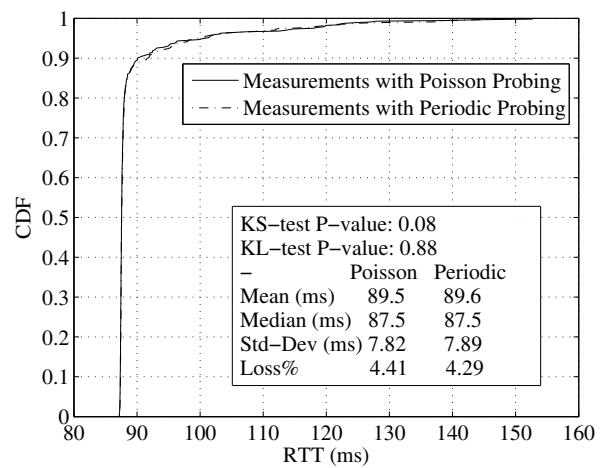

Figure 2: A session in which the KS test performs poorly.

non-parametric goodness-of-fit test can reject $H_{0}$ with a low $P$-value when the two given samples have a statistically significant difference, even if the underlying distributions are strongly non-Gaussian. Recall that P-value is the lowest significance level $\alpha$ at which we can reject the null hypothesis. The P-value is between 0 and $1 . H_{0}$ is often rejected when the P-value is less than 0.05-0.10.

Our initial approach was to use the well-known Kolmogorov-Smirnov (KS) test. This test, however, assumes that the underlying distributions are continuous. Furthermore, it is quite sensitive to that assumption because it focuses on the maximum vertical distance between the two empirical CDFs. It is important to note that the RTT distribution at an Internet path can be almost discontinuous when several probing packets measure the same RTT value that is determined by constant propagation and transmission delays. This discontinuity is located at the minimum (or close to the minimum) measurement, and it is more likely in lightly loaded paths with small queueing delays because the latter can only increase the RTT measurements.

For example, Figure 2 shows the two RTT empirical CDFs measured in a session from MIT to U-Oregon ( $I=20 \mathrm{~ms}, L=480$ bytes). Note that for all practical pur- poses the two distributions are identical. The KS test, however, rejects $H_{0}$ with a low P-value $(P=0.08)$. The reason the test fails is the discontinuity at about $87.5 \mathrm{~ms}$. The maximum vertical distance between the two CDFs is $7.4 \%$, it occurs at the 25-th percentile of the Poisson distribution, while the horizontal offset with the Periodic distribution at that point is only 31 microseconds! It is noted that we observed similar failures with other nonparametric statistical tests, such as the Kruskal-Wallis analysis-of-variance test.

To deal with the previous discontinuity problems, we constructed a more robust non-parametric goodness-offit test based on the Kullback-Liebler (KL) distance, also known as relative entropy [9]. For two discrete probability mass functions (pmf's) $q_{1}$ and $q_{2}$, defined over the same set of values $Q$, the KL distance of $q_{1}$ relative to $q_{2}$ is

$$
D\left(q_{1} \| q_{2}\right)=\sum_{i \in Q} q_{1}(i) \log _{2} \frac{q_{1}(i)}{q_{2}(i)}
$$

It can be shown that $D\left(q_{1} \| q_{2}\right) \geq 0$ and that $D\left(q_{1} \|\right.$ $\left.q_{2}\right)=0$ if and only if the two distributions are identical. Notice that $D\left(q_{1} \| q_{2}\right) \neq D\left(q_{2} \| q_{1}\right)$.

The $K L$ test proceeds in three steps:

1. Estimate the pmf's $s_{e}$ and $s_{p}$ (defined on the same set of bins) from the samples $S_{e}$ and $S_{p}$, respectively. The details of the binning procedure are described in the Appendix.

2. Calculate the KL distance $D\left(s_{e} \| s_{p}\right)$ of the Poisson relative to the Periodic sample.

3. Estimate the distribution of the $\mathrm{KL}$ distance $D\left(s_{e, i} \| \bar{s}_{e, i}\right)$ between randomly chosen partitions $S_{e, i}$ and $\bar{S}_{e, i}$ of the Poisson sample $S_{e}$ ("bootstrapping"). Specifically, suppose that we randomly partition $S_{e}$ in two samples $S_{e, i}$ and $\bar{S}_{e, i}$. Let $s_{e, i}$ and $\bar{s}_{e, i}$ be the corresponding pmf's, and so $D\left(s_{e, i} \|\right.$ $\left.\bar{s}_{e, i}\right)$ is the KL distance of this partition. If we repeat this random partitioning process many times, we can estimate the distribution of $D\left(s_{e, i} \| \bar{s}_{e, i}\right)$.

4. Reject the null hypothesis if $D\left(s_{e} \| s_{p}\right)$ is "too large" relative to the distribution $D\left(s_{e, i} \| \bar{s}_{e, i}\right)$. Specifically, estimate the P-value as

$$
P \approx \operatorname{Prob}\left[D\left(s_{e} \| s_{p}\right) \leq D\left(s_{e, i} \| \bar{s}_{e, i}\right)\right]
$$

and reject $H_{0}$ if $P<0.1$.

Figure 3 shows an example of the distribution of $D\left(s_{e, i} \|\right.$ $\left.\bar{s}_{e, i}\right)$ together with the KL distance $D\left(s_{e} \| s_{p}\right)$ for a particular session. The KL test is more robust than the KS test in the presence of CDF discontinuities. The reason is that instead of relying on a single point of maximum vertical difference, the KL test considers the difference between the two distributions across all bins, weighted by the probability mass at each bin. For the example of 


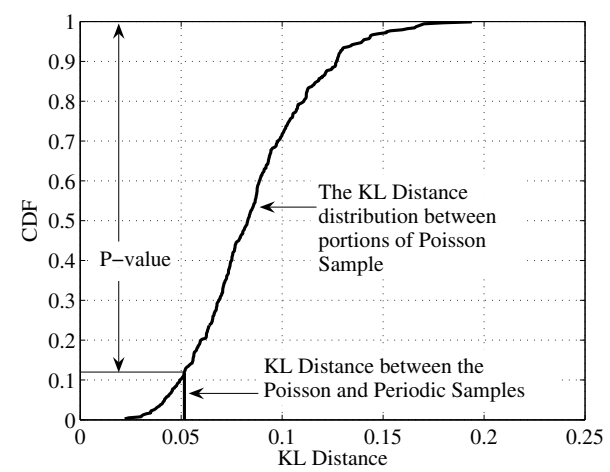

Figure 3: An example of the KL distance distribution for a particular session.

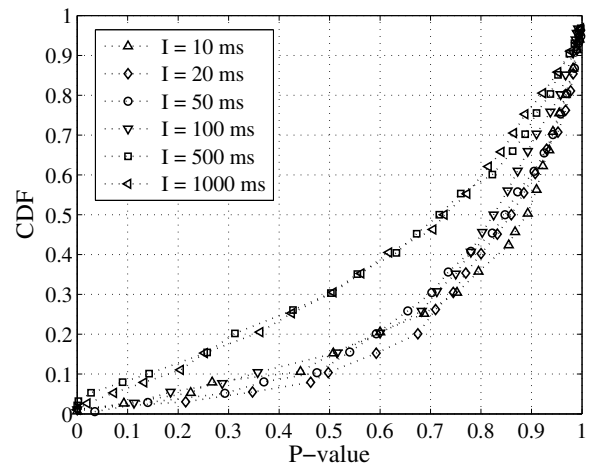

Figure 4: Distribution of P-values for the null hypothesis $H_{0}$, applied to RTT sessions.

Figure 2, the KL test reports a P-value of 0.88 , meaning that the null hypothesis cannot be rejected.

Figure 4 shows the distribution of P-values reported by the KL test for various probing interarrivals $I$. We see that we can reject $H_{0}$ for only $5-10 \%$ of the sessions at a significance level of $10 \%$. The rest of the sessions have significantly higher P-values, meaning that we cannot reject $H_{0}$. So, for more than $90 \%$ of the measurement sessions we can assume that the RTT distributions observed by Poisson and Periodic probing are identical.

Note that the two larger average probing periods, $500 \mathrm{~ms}$ and $1000 \mathrm{~ms}$, result in lower P-values, implying wider (but still not statistically significant) differences between the two RTT distributions. When $I$ is $500 \mathrm{~ms}$ and $1000 \mathrm{~ms}$ the measurement process lasts for $300 \mathrm{sec}$ and $600 \mathrm{sec}$, respectively, and the underlying process shows significant variability during that time window. Consequently, the two probing processes are more likely to observe different values of the underlying process.

\section{Loss rate measurements}

For the loss rate estimates, we used the same set of sessions as in the case of RTTs, except that we now use only the 600 probing packets from the source to the destina-

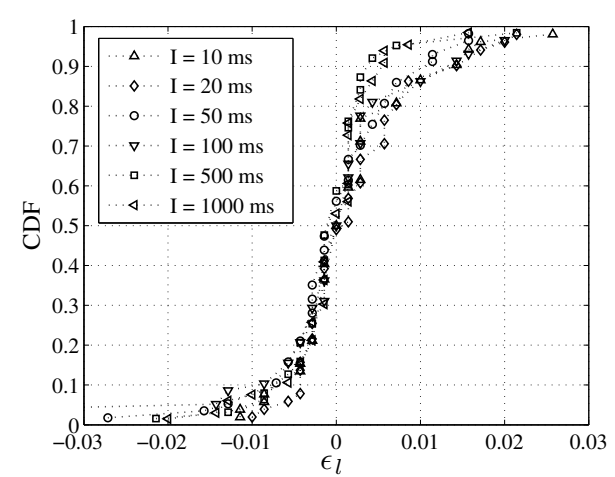

Figure 5: The difference $\epsilon_{l}$ between Poisson and Periodic loss rate estimates.

tion (ignoring the packets in the reverse direction). Let $l_{e}$ and $l_{p}$ be the loss rates, defined as the fraction of lost packets from the Poisson and Periodic streams respectively, in a given session. The difference between the two loss rates is $\epsilon_{l}=l_{e}-l_{p}$. Figure 5 shows the empirical CDF of $\epsilon_{l}$, for the sessions in which we observed some loss, either in Poisson probes, or Periodic probes, i.e., for sessions where $\left(l_{e}+l_{p}\right)>0$, but neither $l_{e}$ or $l_{p}$ exceeds $10 \%$. Note that in about $80 \%$ of the sessions the two loss rates are within $1 \%$, while the maximum loss rate difference is less than $3 \%$.

In theory, we could compare the two loss rates $l_{e}$ and $l_{p}$ using a hypothesis test for the equality of two proportions. Such tests however assume that the underlying loss events are independent, which is not true for Internet losses. Instead, we examine the agreement between Poisson and Periodic probing categorically, classifying the sessions in six classes depending on the loss rate estimated by the Poisson probes. These categories are: lossless $\left(l_{e}=0\right)$, low loss $\left(l_{e} \in(0,1 \%]\right)$, medium loss $\left(l_{e} \in\right.$ $[0.9 \%, 5 \%])$, high loss $\left(l_{e} \in[4.5 \%, 10 \%]\right)$, very high loss $\left(l_{e} \in[9 \%, 20 \%]\right)$, and broken $\left(l_{e} \in[18 \%, 100 \%]\right)$. The categories have a small overlap to avoid boundary effects when examining the agreement between the two probing processes. Note that for this categorization and in the following analysis we include the sessions that have a higher loss rate than $10 \%$.

Figure 6 shows the fraction of sessions in each category (the number at the top of each group). About $78 \%$ of the sessions are classified as lossless or low loss. For each category we also show, with two or three adjacent bars, the fraction of sessions for which the loss rate estimates with Poisson and Periodic probing agree (central bar), as well as the fraction of sessions for which Periodic probing leads to a lower (left bar) or higher (right bar) category. Notice that the two probing techniques agree in more than $70 \%$ of the sessions. Given that the loss process in a congested link can be highly bursty, 


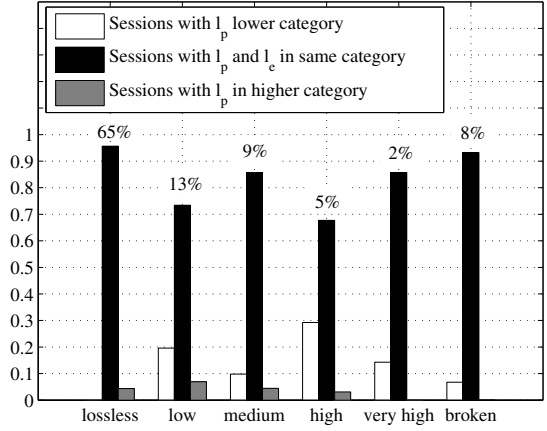

Figure 6: Classification of loss rate estimates in six categories.

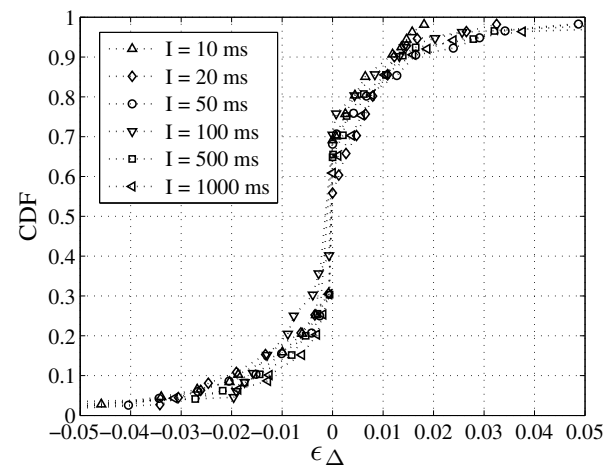

Figure 7: The relative difference $\epsilon_{\Delta}$ between Poisson and Periodic dispersion medians.

and since our sample size is rather small to accurately estimate low loss rates, it is not surprising that about 10$30 \%$ of the sessions observe different loss categories with Poisson and Periodic probing.

\section{Packet pair dispersion measurements}

\section{Comparison of median dispersions:}

In the dispersion measurements, we send back-to-back packet pairs of size $L$ from the source to the destination. The latter measures the time spacing ("dispersion") $\Delta$ between the arrival of the first and the second packet. The dispersion at the destination is related to the cross traffic load and available bandwidth in the network path [4]. Specifically, the higher the cross traffic load is at the path's bottleneck, the wider the dispersion of the packet pairs at the destination due to the interference of cross traffic between the probing pair.

For these measurements, if one of the two packets is lost, the corresponding pair is ignored. Note that the average probing interarrival $I$ controls the time spacing between successive packet pairs, not between packets of the same pair, which are always sent back-to-back.

Let $\Delta_{e}$ and $\Delta_{p}$ be the median dispersions estimated

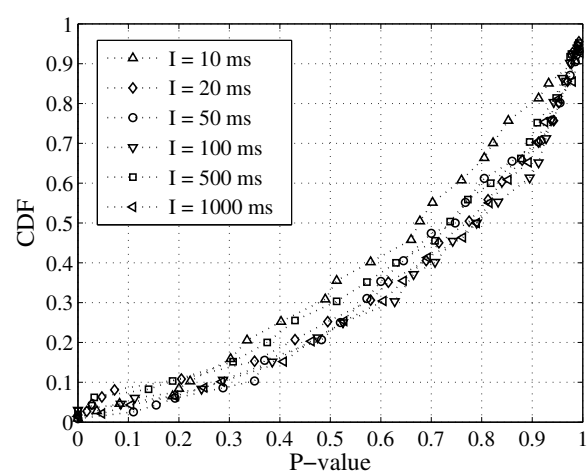

Figure 8: Distribution of P-values for the null hypothesis $H_{0}$, applied to dispersion sessions.

from the Poisson and Periodic probing streams, respectively, for a particular session. We define the relative difference between the two dispersion medians as $\epsilon_{\Delta}=2 \frac{\Delta_{e}-\Delta_{p}}{\Delta_{e}+\Delta_{p}}$. Figure 7 shows the empirical CDF of $\epsilon_{\Delta}$. Note that about $90 \%$ of the sessions have $\left|\epsilon_{\Delta}\right|<2.5 \%$, while the relative difference does not exceed $8 \%$. Consequently, as in the case of RTTs, we see that the Poisson and Periodic probing processes estimate practically the same dispersion, at least in terms of a point estimate for the first moment.

Goodness-of-fit test: We also examined whether the two probing processes observe the same dispersion distribution. To do so, we used again the KL test described in $\S 3$. This time the null hypothesis $H_{0}$ is that the two dispersion samples (Poisson and Periodic) for a given session follow an identical distribution. Figure 8 shows the distribution of P-values reported by the KL test, for various probing interarrivals $I$. Notice that the $\mathrm{P}$-value is less than $10 \%$ for only $5-10 \%$ of the sessions. The rest of the sessions have significantly higher P-values, implying that we can assume $H_{0}$ to be true.

\section{Discussion}

The experimental results in this paper indicate that there may not be a significant difference between Poisson and Periodic probing, at least in the context of real Internet measurements. This does not mean that we recommend the use of Periodic probing over Poisson probing. We note however that measurement studies that use, or have used, Periodic probing should not be dismissed based on that fact, and they may also have practical benefits compared to Poisson probing. A few additional remarks on the accuracy of Poisson and Periodic probing follow.

First, it is important to note that the fraction of dropped Poisson probing packets at a network queue does not estimate the packet loss rate, i.e., the fraction of dropped packets among all arrived packets; instead, it estimates the fraction of time that the queue is full. The latter is 
equal to the loss rate in a queue with Poisson packet arrivals; this follows applying the PASTA property to all packets (not only the probing packets). For more bursty traffic, however, the packet loss rate can be higher than the fraction of time that the queue is full. Consequently, even if Poisson and Periodic probing observe the same loss rate, that fraction should not be expected to be equal to the underlying loss rate.

Second, Periodic probing at a certain interarrival $I$ cannot "see" effects that occur in lower time scales (or higher frequency). In the case of loss rate estimation, in particular, loss events in Drop-Tail queues can be very bursty. If the duration of loss bursts is much lower than $I$, then Periodic probing may underestimate both the fullqueue probability and the loss rate. Poisson probing, however, with a sufficiently large number of samples, should be able to estimate the full-queue probability.

Third, it is important to note that even if we generate a Poisson probing stream at the source, the probing packets may not arrive at the bottleneck link as a Poisson process. Consider, for instance, that the output of an $M / D / 1$ queue is not a Poisson process. In more practical terms, if the probing packets go through a storeand-forward link with capacity $C$, then their interarrivals after that link cannot be lower than $L / C$, where $L$ is the packet size. Consequently, the probing packets will no longer be a Poisson stream and PASTA will not apply. This issue is important for Internet measurements, given that most network paths go through multiple queues.

\section{Appendix: Density estimation}

Suppose that we are given two samples $S_{1}$ and $S_{2}$ that take values in a range $R_{S}$. We want to approximate the probability density functions of the two samples with the probability mass functions $q_{1}(i)$ and $q_{2}(i)$ defined over a set of bins $Q$, such that $q_{1}(i)$ and $q_{2}(i)$ is the fraction of measurements in the $i^{\prime}$ th bin from $S_{1}$ and $S_{2}$, respectively, and $\sum_{i \in Q} q_{1}(i)=\sum_{i \in Q} q_{2}(i)=1$. We select the initial bin size $w$ based on the Freedman-Diaconis rule as $w=2 n^{-1 / 3} \mathrm{IQ}$, where $n$ is the number of samples in the joint sample $S_{1} \cup S_{2}$ and IQ is the interquartile range of $R_{S}$ [10]. We then proceed to determine the bin boundaries, with the first bin placed based on the minimum measurement, and to estimate the functions $q_{1}(i)$ and $q_{2}(i)$.

The problem, however, is that some bins may not include enough measurements from each sample. When that is the case we are not able to accurately estimate the "likelihood ratio" $\log _{2} \frac{q_{1}(i)}{q_{2}(i)}$ of Equation 2. To guarantee that each bin contains at least a certain number of measurements from both samples, we use an adaptive histogram approach. Specifically, if a bin does not include at least $1 \%$ of the measurements from each sample, we merge that bin with the bin at its right. The process is repeated until the previous constraint is met. If there are no measurements from that sample at any of the right bins, we merge the problematic bin with the bin at its left. In practice, we found that the previous heuristic is quite robust, as long as the two samples take values over approximately the same range.

\section{Acknowledgments}

We are grateful to Prof. Paul Kvam (ISYE, Georgia Tech) for suggesting the use of relative entropy in a goodness-of-fit test. This work was supported in part by the NSF CAREER award ANIR-0347374, NSF award ANI-024085, and by a gift from DoCoMo USA Labs.

\section{References}

[1] V. Paxson, G.Almes, J.Madhavi, and M.Mathis, Framework for IP Performance Metrics, May 1998, RFC 2330.

[2] V. Paxson, Measurements and Analysis of End-toEnd Internet Dynamics, Ph.D. thesis, University of California, Berkeley, Apr. 1997.

[3] Y. Zhang, N. Duffield, V. Paxson, and S. Shenker, "On the Constancy of Internet Path Properties," in Proceedings of ACM/USENIX Internet Measurement Workshop (IMW), Nov. 2001, pp. 197-211.

[4] J. Strauss, D. Katabi, and F. Kaashoek, "A Measurement Study of Available Bandwidth Estimation Tools," in Proceedings of ACM/USENIX Internet Measurement Conference (IMC), 2003.

[5] D. Papagiannaki, S. Moon, C. Fraleigh, P. Thiran, F. Tobagi, and C. Diot, "Analysis of Measured Single-Hop Delay from an Operational Backbone Network," in Proceedings of IEEE INFOCOM, 2002.

[6] R. Caceres, N. G. Duffield, J. Horowitz, and D. Towsley, "Multicast-Based Inference of Network-Internal Loss Characteristics," IEEE Transactions in Information Theory, pp. 24622480, 1999.

[7] R. Wolff, "Poisson Arrivals See Time Averages," Operations Research, vol. 30, no. 2, pp. 223-231, 1982.

[8] J. Sommers, P. Barford, N. G. Duffield, and A. Ron, "Improving Accuracy in End-to-End Packet Loss Measurements," in Proceedings ACM SIGCOMM, Aug. 2005.

[9] M. Lexa, "Useful Facts about the Kullback-Leibler Discrimination Distance," Tech. Rep., Rice University, 2004.

[10] D. Scott, Multivariate Density Estimation: Theory, Practice and Visualization, Prentice Hall, 1992. 